\title{
PONDERMOTIVE ACCELERATION OF IONS BY RELATIVISTICALLY SELF-FOCUSED HIGH-INTENSITY SHORT PULSE LASER *
}

\author{
$\underline{\text { A. Maksimchuk }}^{\dagger}$, S. Gu, K. Flippo, S.-Y. Chen and D. Umstadter ${ }^{\ddagger}$, \\ G. S. Sarkisov ${ }^{\S}$, V. Yu. Bychenkov, V. N. Novikov and V. T. Tichonchuk
}

Abstract

We report on the observation of high energy ions with energies up to $1 \mathrm{MeV}$ accelerated pondermotively by a relativistically self-focused intense $5 \mathrm{TW}, 400$ fs laser pulse in a supersonic $\mathrm{He}$ gas jet on the distance of a laser spot size. Probing interferometry was used to observe on-axis electron-ion cavitation followed by the plasma expansion with a high radial velocity of $\sim 3.8 \cdot 10^{8} \mathrm{~cm} / \mathrm{s}$. Using the nuclear track detector CR-39, we confirmed that the ions are preferentially accelerated in the radial direction and the total energy in high energy ions is about $1 \%$ of the laser energy. Developed kinetic modeling provides a reasonable description of a plasma channel formation and ion acceleration.

\section{INTRODUCTION}

An interaction of ultra-high intensity short laser pulses with underdense plasma is of considerable interest from the standpoint of basic physics and potential application for advanced inertial confinement fusion, $x$-ray lasers and particle accelerators $[1,2,3]$. The ultra-high electromagnetic fields in the laser focus produce an extremely high pondermotive force that expels free electrons from the laser axis and relativistically modifies the electron mass, plasma frequency and the plasma refractive index so that the plasma acts as a positive lens that further increases the focused intensity . If the laser pulse duration is long enough, the charge separation produces strong electrostatic fields $\sim 1 \mathrm{GV} / \mathrm{cm}$ which can accelerate ions to $\mathrm{MeV}$ energies.

\section{EXPERIMENTAL SETUP}

The experiment has been performed using the $10 \mathrm{TW}$ Ti:sapphire-Nd:glass CPA laser system at the Center for Ultrafast Optical Science, University of Michigan. The laser operates at the wavelength $\lambda_{0}=1.053 \mu \mathrm{m}$, and produces pulses with the energy up to $4 \mathrm{~J}$ in $400 \mathrm{fs}$ (FWHM) with an intensity contrast ratio $\sim 10^{5}: 1$. The $50 \mathrm{~mm}$ diameter laser beam was focused with an off-axis parabolic mirror $(f / 3.3, f=16.5 \mathrm{~cm})$ to a $10 \mu \mathrm{m}$ spot with

\footnotetext{
${ }^{*}$ We acknowledge the support of the Division of High Energy Physics, Office of Energy Research, U. S. Department of Energy, the National Science Foundation and Russian Foundation for Basic Research.

† Email: tolya@umich.edu

$\ddagger$ Center for Ultrafast Optical Science, University of Michigan, Ann Arbor, MI 48109.

$\S$ Current address Department of Physics, University of Nevada, Reno, NV 89557.

ฯP. N. Lebedev Physics Institute, Russian Academy of Sciences, Mo-
} scow. a vacuum intensity of $6 \cdot 10^{18} \mathrm{~W} / \mathrm{cm}^{2}$. The radial distribution of laser intensity demonstrates also $\sim 50 \mu \mathrm{m}$ in diameter wings that contain approximately $10 \%$ of laser energy. The laser beam was focused in a high backing pressure $(\sim 7 \mathrm{MPa}) \mathrm{He}$ gas jet expanding through a $1 \mathrm{~mm}$ diameter nozzle. The optimal conditions for beam guiding correspond to the laser focusing on the jet edge at a distance $x=0.5-0.75 \mathrm{~mm}$ from the nozzle, where the $\mathrm{He}$ atom density is $\sim 4 \cdot 10^{19} \mathrm{~cm}^{-3}$.

The probe beam with wavelength $\lambda=1.053 \mu \mathrm{m}$ was obtained by splitting $\sim 5 \%$ of the pump pulse, propagated through an adjustable motorized optical delay, and probed the plasma in the direction perpendicular to the interaction beam. The plasma was imaged by spherical lens with the angular aperture $7^{\circ}$ on two 12-bit CCD cameras to produce shadowgram and interferogram. The spatial and temporal resolution was $10 \mu \mathrm{m}$ and $400 \mathrm{fs}$, respectively. The airwedge shearing interferometer has been used for the electron density measurements. To measure ion angular distribution we placed around the gas jet at the distance $\sim 16$ $\mathrm{cm} 11$ slits with a width $\sim 100 \mu \mathrm{m}$ and $\sim 10$ degrees apart from each other. We positioned nuclear track detectors CR39 behind the slits at a distance $\sim 16 \mathrm{~cm}$. Analysis of ion energy distribution was done by measurements of an individual track size in CR-39 after an etching procedure.

\section{RESULTS AND DISCUSSION}

We followed the plasma evolution for the time period from -2 to +55 ps. We observed a fast gas ionization in the cone angle $\left(\sim 17^{\circ}\right)$ of the laser $\sim 2$ ps before the pump pulse arrival. The laser intensity in the temporal foot and in the spatial wings of the pulse was above $10^{15} \mathrm{~W} / \mathrm{cm}^{2}$, that was sufficient for the tunneling ionization of $\mathrm{He}$ atoms. The transverse size of the formed plasma was about $100 \mu \mathrm{m}$ in diameter. When the laser power exceeds a critical power $P_{c}=17 n_{c} / n_{e} \mathrm{GW}$ (where $n_{c}$ is the critical density for the interaction laser pulse) self-focusing and self-channeling can occur as a result of two effects: (i) the relativistic increase of electron mass in the laser field and (ii) the reduction of the electron density on axis due to the expulsion of the electrons by the laser pondermotive force. We observed the first signature of plasma channel formation at $t=0 \mathrm{ps}$. The change in sign of the phase shift derivative in a narrow axial region in the interferogram indicates the decrease of the phase shift and hence the decrease of on-axis electron density Fig. 1.

The two-dimensional reconstruction of the electron density profile has been performed using the Abel inversion procedure with an assumption of the axial symmetry of a 


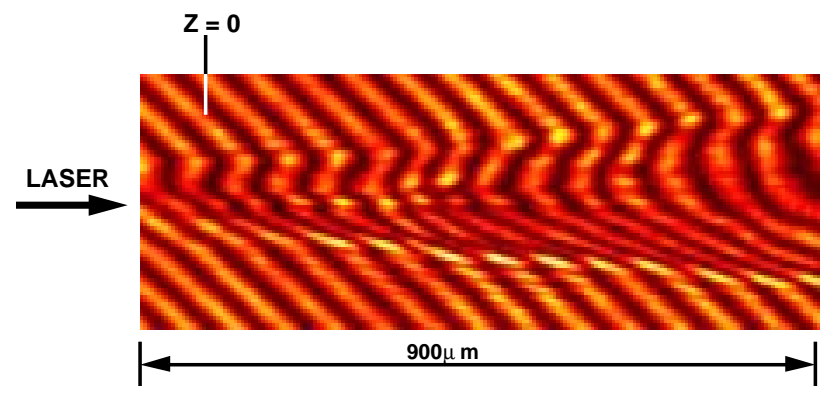

Figure 1: Interferogram taken at time $\mathrm{t}=5 \mathrm{ps}$, showing onaxis electron cavitation. Plane $z=0$ indicates the position of focal plane in vacuum.

plasma and attributing all changes of the refraction index $\mu$ to free electrons. The inferred electron density profile for time moment $t=35 \mathrm{ps}$ is presented in Fig. 2. The maximum electron density is $\sim 7.6 \cdot 10^{19} \mathrm{~cm}^{-3}$ at a radius of $\sim 20 \mu \mathrm{m}$ and the estimated depth of the plasma channel is up to $80-90 \%$. The radius of the plasma channel is less than $10 \mu \mathrm{m}$.

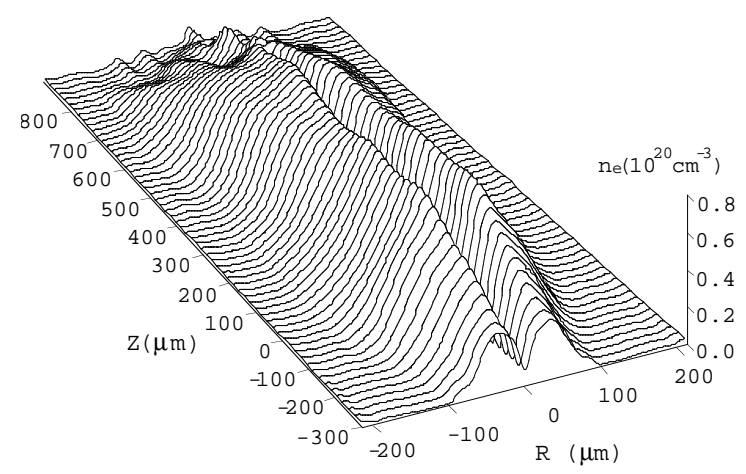

Figure 2: Spatial electron density distribution for time moment of $\mathrm{t}=35 \mathrm{ps}$.

We also observed fast ambient gas ionization in the radial direction for probe time delays greater than $10 \mathrm{ps.} \mathrm{If}$ we define the plasma edge as a region where the electron density is equal to $5 \cdot 10^{18} \mathrm{~cm}^{-3}$, then from $R-t$ diagram of plasma expansion, presented at Fig. 3 one can find, that the radial expansion velocity is equal to $\sim 3.8 \cdot 10^{8} \mathrm{~cm} / \mathrm{s}$. Plasma regions with a higher degree of ionization, $1.5 \cdot 10^{19}$ $\mathrm{cm}^{-3}$ expand with velocity $\sim 2.5 \cdot 10^{8} \mathrm{~cm} / \mathrm{s}$. The channel diameter has even slower velocity $\sim 4 \cdot 10^{7} \mathrm{~cm} / \mathrm{s}$.

We attributed an ambient gas ionization to the propagation of fast Helium ions. These ions are accelerated by the electrostatic field of charge separation produced in a plasma when the electrons are expelled from the laser beam channel due to the radial component of the ponderomotive force and have an energy of more than $300 \mathrm{keV}$. The mean free path of such ions in a normal pressure gas is few millimeters, and they penetrate through a gas as a projectiles, experiencing only small angle elastic scattering and loosing their energy to the neutral atoms via excitation and ioniza-

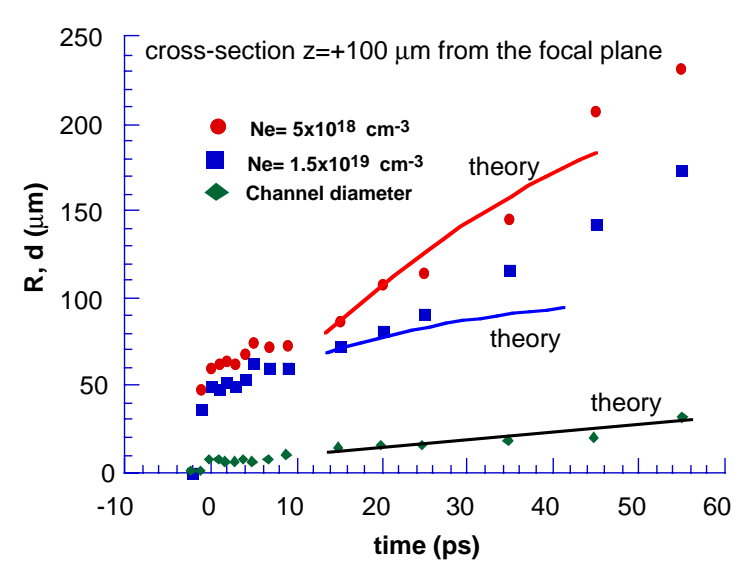

Figure 3: Evolution of the plasma radius for the crosssection $z=100 \mu \mathrm{m}$. Experimental points show the positions of electron densities $5 \cdot 10^{18} \mathrm{~cm}^{-3}$ and $1.5 \cdot 10^{19} \mathrm{~cm}^{-3}$, and the evolution of the plasma channel diameter. The theoretical curves have been obtained from the developed kinetic model.

tion. The velocity of such fast ions is comparable to the velocities of bound electrons in the Helium atom, and therefore ions efficiently ionize the ambient gas. Their ionization cross-section is about $3 \cdot 10^{-16} \mathrm{~cm}^{2}$ [4], and, therefore, each ion produces approximately one electron-ion pair on a $1 \mu \mathrm{m}$ path in the Helium gas with a density of $\sim 4 \cdot 10^{19}$ $\mathrm{cm}^{-3}$.

To formulate quantitatively the physical model of plasma channel formation we supplement the quasistationary description of the relativistic electrons by the kinetic description of the cold collisionless nonrelativistic ions with the mass $m_{i}$ and charge $Z e$. Assuming that ions move only in radial direction, the kinetic equation for the ion distribution function, $f_{i}$, reads:

$$
\frac{\partial f_{i}}{\partial t}+v_{i} \frac{\partial f_{i}}{\partial r}+\frac{Z}{m_{i}} F_{p} \frac{\partial f_{i}}{\partial v_{i}}=0 .
$$

The last term in the left hand side accounts for the ion acceleration due to the electric field produced by the ponderomotive force:

$$
\mathbf{F}_{p}=-m_{e} c^{2} \nabla \sqrt{1+a^{2} / 2},
$$

where $a=0.85 \cdot 10^{-9} \lambda_{0}[\mu \mathrm{m}] \sqrt{I\left[\mathrm{~W} / \mathrm{cm}^{2}\right]}$ is the normalized slowly varying vector-potential of the laser field and $I$ is the laser pulse intensity. Equation (1) has been solved numerically by the particle-in-cell method. The radial velocity of the ions after the laser pulse ends reads:

$$
v_{i}=-\frac{Z m_{e}}{m_{i}} c^{2} \frac{\partial}{\partial r} \int d t \sqrt{1+a^{2} / 2} .
$$

This formula predicts the ion energy spectrum $0<\epsilon_{i} \leq$ $\epsilon_{\max }$ with the energy cutoff

$$
\epsilon_{\max } \sim \frac{Z^{2} m_{e}^{2} c^{4}}{16 m_{i} r_{0}^{2}} \frac{a_{0}^{4} \tau^{2}}{1+a_{0}^{2} / 2} \approx 3 \frac{Z^{2}}{A} \frac{\tau^{2}}{r_{0}^{2}} \frac{a_{0}^{4}}{1+a_{0}^{2} / 2} \mathrm{MeV},
$$


where $a_{0} \approx 3.6 \sqrt{E_{0} / \tau} \lambda_{0} / r_{0}$ is the maximum vectorpotential of the interaction laser pulse and $A$ is the ion mass number. Here $E_{0}$ is measured in Joules, $\tau$ in ps, $\lambda_{0}$ and $r_{0}$ in microns. According to this formula, in the relativistic limit, $a_{0} \geq 1$, the ion energy is approximately proportional to the pulse energy and pulse length, and inversely proportional to the fourth power of the laser beam radius. This high power radius dependence can be used to resolve an inverse problem: to estimate the laser channel radius knowing the ion energy.

The laser channel radius, $r_{0}$ has been varied in order to fit the observed rate of secondary plasma production, $d N_{e} / d t$, and the velocity of plasma expansion with a given electron density (see Fig. 3). We found also an importance of the He-gas excitation which can contribute as much as $50 \%$ to the observed interferometrically change in the plasma refraction index. The comparison of axial velocities of plasma expansion calculated from the model with the measured experimentally allows to deduce an axial dependence of the laser channel radius, $r_{0}(z)$. Knowing the laser energy in a given cross-section, one finds also the onaxis laser intensity, $I_{0}(z)$. In this simulation we assume an exponential decrease of $E_{0}$ with $z$ that corresponds to $60 \%$ laser energy loss on $1 \mathrm{~mm}$ length and was measured in other experiment [5]. The results of this reconstruction of the laser channel characteristics in a plasma are shown in Fig. 4. One can see two regions of laser channel compression at $z=100$ and $600 \mu \mathrm{m}$. We infer that the maximum value of laser intensity in the plasma channel exceeds the vacuum intensity by almost 3 times.

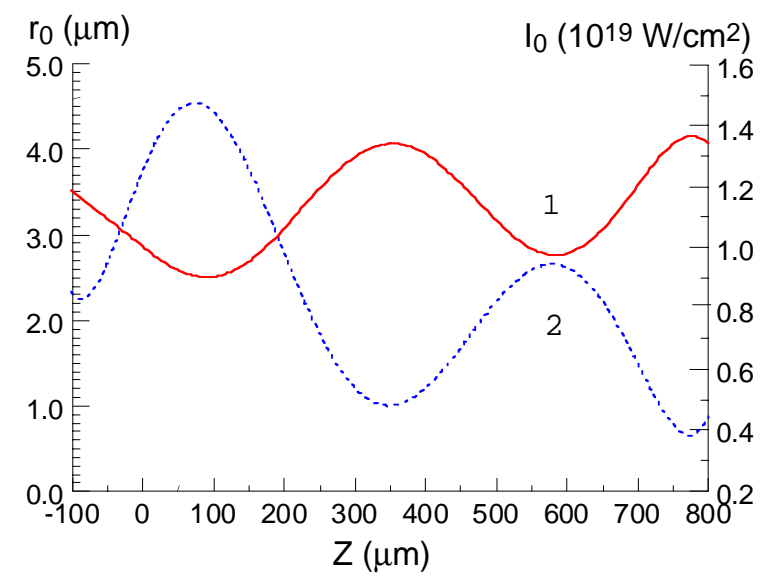

Figure 4: Axial dependence of the laser channel radius (curve 1, solid line) and on-axis laser intensity (curve 2, dashed line) for parameters of the experiment.

Knowing the axial dependence of the laser energy, $E_{0}(z)$, and the laser channel radius, $r_{0}(z)$, we can find the axial characteristics of fast ion generation. In Fig. 5a we show the total number of fast ions, with energies above the given energy, $\epsilon_{i}$, integrated over the laser channel length: $N_{\text {total }}=\int d z \int_{\epsilon_{i}}^{\epsilon_{\max }} d \epsilon N_{i}(\epsilon, z)$. We found that the total number of the fast ions with the energy higher than 100
$\mathrm{keV}$ is $\sim 10^{12}$ and with the energy higher than $300 \mathrm{keV}$ is $\sim 3 \cdot 10^{11}$. This results were confirmed by the direct observation of high energy ions with the nuclear track detector CR-39. We also found when studying the angular distribution of the emitted ions that they are accelerated preferentially in the direction perpendicular to the channel axis. The FWHM of the ion angular distribution (see Fig. 5b) is $\sim 20$ degrees which is close to the cone angle of the focused beam. We didn't observed any accelerated ions in a forward direction because of much smaller pondermotive force in this direction.
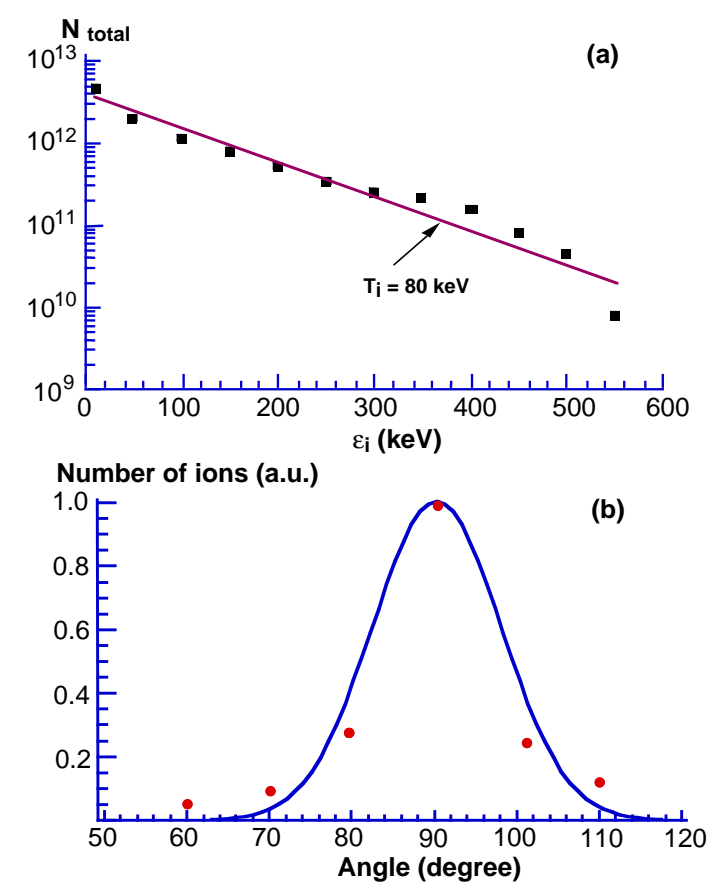

Figure 5: (a) Total number of fast ions with energies exceeded $\epsilon_{i}$ integrated over the laser channel length for parameters of the experiment. Solid line is the Maxwellian function with the temperature of $80 \mathrm{keV}$. (b) Normalized angular distribution of fast ions emitted from the channel. Solid line is a Gaussian fit to the data.

An estimated total energy in fast ions can be as high as one percent of the laser energy. In spite of predicted by the theory a sharp energy cut-off at about $500 \mathrm{keV}$ we observed $\alpha$-particles with the energy up to $1 \mathrm{MeV}$. This result can be explained by the influence of electron pressure due to hightemperature plasma heating which has not been taken into consideration in a theoretical modeling.

\section{REFERENCES}

[1] M. Tabak et. al., Phys. Plasmas 1, 1626 (1994).

[2] J. Dunn et. al., Phys. Rev. Lett. 80, 2825 (1998).

[3] D. Umstadter et. al., Science 273, 472 (1996).

[4] M. E. Rudd et. al., Phys. Rev. A 32, 2128 (1985).

[5] S.-Y. Chen et al., Phys. Rev. Lett. 80, 2910 (1998). 